\title{
Prévalence de la contamination par Salmonella des élevages de poulet de chair au Maroc
}

\author{
Abdellah Chaiba ${ }^{1,2,{ }^{*}}$ et Fouzia Rhazi Filali ${ }^{1}$ \\ ${ }^{1}$ Département de biologie, faculté des sciences de Meknès, Meknès, Maroc \\ 2 SADS-CRMEF Souss Massa Daraa Inzegane, Agadir, Maroc
}

\begin{abstract}
Résumé - L'objectif de la présente étude est d'évaluer l'impact des pratiques de gestion et de l'hygiène générale sur la contamination des élevages en bande de poulets de chair par Salmonella à Meknès (Maroc). L'étude a été menée de septembre 2005 à octobre 2007 sur un échantillon de 25 élevages de poulets de chair. Un questionnaire a été soumis aux éleveurs et des échantillons de fientes fraîches de poulets ont été collectés. Les résultats indiquent que $24 \%$ des élevages sont infectés par Salmonella spp. Onze facteurs de risque sur 24 testés sont significativement associés à la contamination des lots d'élevage par Salmonella. Les facteurs de risque de l'infection sont spécifiquement liés au manque d'hygiène. La contamination de la bande précédente et des poussins d'un jour augmente le risque d'infection par Salmonella. Cependant, l'utilisation d'antibiotiques à la mise en place des poussins et l'usage de détergent pour le nettoyage diminuent ce risque.
\end{abstract}

Mots clés : Maroc / poulet / prévalence / Salmonella spp.

\begin{abstract}
Prevalence of Salmonella infection of broiler-chicken farms in Morocco. The aim of the present study was to assess the association of managerial practices and general hygiene and their impact on Salmonella infection of 25 broiler farms around Meknès (Morocco). A questionnaire was submitted to the farmers from September 2005 to October 2007 and samples of fresh broiler dropping were taken. Results indicate that $24 \%$ of the farms are infected by Salmonella spp.; 11 risk factors out of the 24 tested are associated with Salmonella infection of the farm. The specific risk factors for infection of broiler farms are linked to inadequate hygienic measures. Salmonella infection of the previous flock and of day-old chicks increases the risk of Salmonella infection. Using antibiotics on day-old chicks and a detergent for cleaning decreases the risk of contamination.
\end{abstract}

Keywords: broiler-chicken / Morocco / prevalence / Salmonella spp.

\section{Introduction}

Depuis plusieurs décennies, les toxi-infections d'origine alimentaire constituent la cause la plus fréquente de maladies intestinales chez l'homme dans la plupart des pays. Parmi les bactéries impliquées, Salmonella et Campylobacter sont à l'origine de plus de $90 \%$ des cas signalés de toxi-infections alimentaires d'origine bactérienne dans le monde (Thorns, 2000).

Parmi les sources de contamination identifiées, la principale est l'ingestion d'aliments contaminés, en particulier l'eau, le lait cru ou la viande insuffisamment cuite (Kimura et al., 2004) et les contaminations croisées qui en résultent. La viande de volaille est un vecteur important de la transmission, responsable de la plupart des cas humains de salmonelloses (Davies et al., 2001).

Il est essentiel de prendre en considération le problème de la contamination des élevages (Bailey et al., 2001), tant pour son

\footnotetext{
*Auteur de correspondance : abchaiba@yahoo.fr
}

impact sur la santé publique que pour les répercussions économiques non négligeables qu'il peut engendrer. Or, si la contamination de la viande est possible à tous les niveaux de la chaîne de production (Mead, 1993), la période d'élevage représente une étape critique pour le développement de la bactérie Salmonella. La connaissance des modalités de contamination des poulets de chair par ce germe au cours de cette période est donc primordiale afin d'empêcher son développement tout au long de la filière.

Pour répondre au besoin accru en protéines animales, le secteur avicole marocain est en plein essor. Cependant, cette expansion de la production a eu lieu sans la maîtrise systématique de l'hygiène tout au long de cette filière. Jusqu'à présent, aucune étude n'a été faite au Maroc sur les facteurs de contamination des élevages de poulet de chair par Salmonella. C'est dans ce but que nous avons réalisé ce travail d'identification et de quantification de facteurs (surtout relatifs à la conduite de l'élevage et aux mesures sanitaires pratiquées) contribuant à la colonisation par Salmonella des élevages de poulet de chair dans la région de Meknès (Maroc). Cela nous a 
Tableau 1. Organisation des prélèvements dans les élevages de poulets de chair.

Table 1. Sampling protocol in broiler-chicken farms.

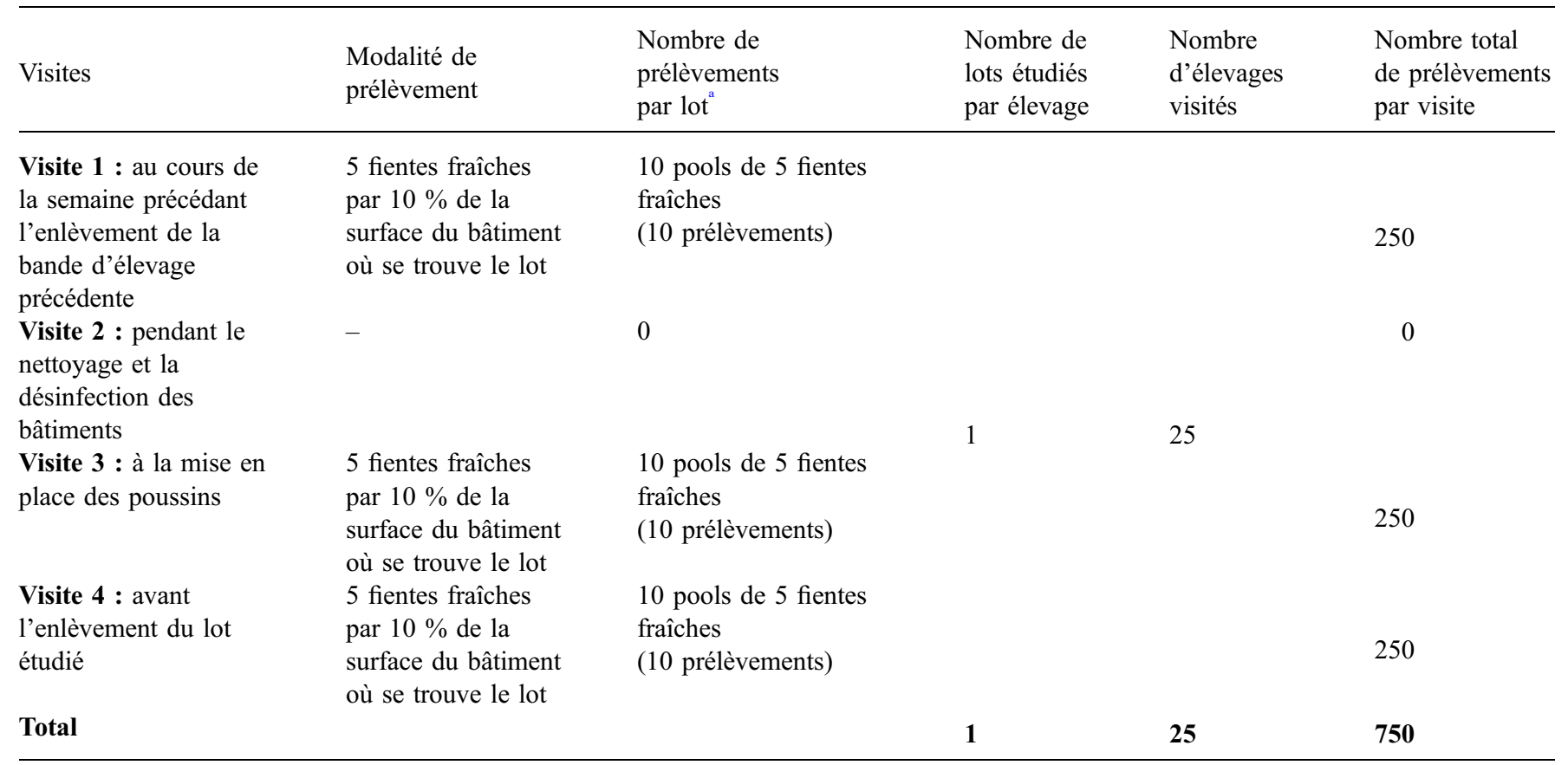

${ }^{a}$ Un lot correspond à un ensemble de poulets de même âge et placés dans un lieu délimité (bâtiment d'élevage) à l'intérieur duquel ils sont libres de se déplacer.

permis de prioriser les moyens de lutte à mettre en œuvre pour diminuer la prévalence de cette bactérie. Les facteurs décrits sont relatifs à la conduite de l'élevage et aux mesures sanitaires pratiquées dans les bâtiments d'élevage.

\section{Matériel et méthode}

\section{1 Échantillon étudié}

L'étude a été menée de septembre 2005 à octobre 2007 sur un échantillon de 25 élevages en bande de poulets de chair situés dans la région de Meknès. La sélection de ces élevages a été fondée sur la volonté du propriétaire de coopérer au cours de l'étude. Nous avons essayé de couvrir toutes les communes, avec au minimum un élevage par commune (Meknès, Boufekrane, Agouray et Elhajeb).

Quatre visites ont été effectuées dans chaque élevage : au cours de la semaine précédant l'enlèvement de la bande d'élevage précédente, pendant le nettoyage et la désinfection des bâtiments, à la mise en place des poussins, et avant l'enlèvement de la bande d'élevage étudiée (34 à 45 jours d'âge). Un seul lot par élevage a fait l'objet de cette étude.

\subsection{Collecte des données}

Afin de déterminer la contamination des poulets par Salmonella, des prélèvements ont été effectués lors de trois visites (Tab. 1). Par élevage par visite, dix pools de cinq fientes fraîches étaient prélevés dans des pots stériles. Ce prélèvement était fait de façon à balayer la plus grande surface du bâtiment. En parallèle, un questionnaire rempli par un technicien des services vétérinaires avec l'éleveur permettait de récolter des données relatives à la conduite de l'élevage, aux mesures sanitaires pratiquées, à l'aménagement et à l'entretien du bâtiment d'élevage. Ce questionnaire a été testé dans une étude préliminaire réalisée dans cinq élevages. Dans sa version finale, il est composé de 76 questions dont 93,42\% sont fermées.

\subsection{Analyse bactériologique des échantillons}

L'analyse bactériologique est menée selon la norme AFNOR en vigueur (NF U : 47-100). Vingt-cinq grammes de chaque échantillon sont pré-enrichis dans $225 \mathrm{ml}$ d'eau peptonée tamponnée (AES laboratoire, Combourg, France) et incubés pendant 20 heures à $37^{\circ} \mathrm{C}$. Deux millilitres du milieu de pré-enrichissement sont ensemencés dans $20 \mathrm{ml}$ de bouillon au tétrathionate de Müller-Kauffmann (MK) et $100 \mu$ l sont ensemencés à la surface d'une boîte de Petri contenant le milieu MSRV (Modified Semi-Solid Rappaport Vassiliadis) (Merck, Nogent-sur-Marne, France). Ces deux milieux de culture sélectifs sont incubés respectivement à $42^{\circ} \mathrm{C}$ pendant 24 heures et $41,5^{\circ} \mathrm{C}$ pendant 48 heures. Les isolements sont réalisés, d'une part, sur milieu de Salmonella-Shigella (SS) (Humeau, La Chapelle-sur-Erdre, France) à partir d'un halo de migration caractéristique sur milieu MSRV et, d'autre part, sur gélose $\mathrm{XLT}_{4}$ (xylose-lysine-tergitol 4) (AES laboratoire, Combourg, France) pour chaque bouillon de $\mathrm{MK}$. Après incubation à $37^{\circ} \mathrm{C}$ pendant 24 heures, les colonies caractéristiques de Salmonella ont été confirmées à partir de critères biochimiques sur les 
milieux suivants : Kligler-Hajna (AES laboratoire, Combourg, France), citrate de Simmons, mannitol-mobilité, urée-indole, Clark et Lubs, malonate, ornithine décarboxylase, lysine décarboxylase, arginine dihydrolase, arabinose, inositol et adonitol.

\subsection{Traitements statistiques des données}

L'unité d'étude est le lot visité infecté si au moins un pool de fientes est testé positif en Salmonella. Un test de $\mathrm{Khi}^{2}$ (Statview) à $5 \%$ a été effectué dans le but de tester les liaisons entre chaque variable explicative et la variable «présence/ absence de Salmonella ». Pour le calcul des odds ratios (OR) avec un intervalle de confiance à $95 \%$ et le risque relatif (RR), nous avons utilisé le logiciel Statistica 6.0 (Statsoft Ltd., Chicago, Illinois).

\section{Résultats}

\subsection{Caractéristiques des bâtiments d'élevage}

Les résultats obtenus à travers le questionnaire nous ont permis de connaître les 25 élevages où les fientes ont été prélevées. Le sol du bâtiment est constitué dans $32 \%$ des élevages de terre battue, les autres sont bétonnés. Soixante-huit pour cent des fermes visitées regroupent plusieurs bâtiments d'élevage distants de moins de $500 \mathrm{~m}$ les uns des autres. Les alentours des poulaillers sont cimentés et comportent des fossés de drainage dans près de la moitié des cas (Tab. 2). $28 \%$ des bandes d'élevage précédant les lots étudiés sont infectées par Salmonella.

Cinq élevages parmi les 25 étudiés n'ont pas de clôture et donnaient libre accès aux animaux (chiens et chats...). L'équipement se limite au strict minimum (mangeoires, abreuvoirs et radiants de chauffage) avec inexistence de systèmes de ventilation et d'isolation pour quatre élevages sur 25 . L'absence d'hygromètre dans $24 \%$ des élevages pose des problèmes de maîtrise des conditions ambiantes, notamment en saison chaude.

\subsection{Les animaux}

À l'abattage, les animaux sont âgés de 40 jours en moyenne et pèsent entre 0,9 et $2,25 \mathrm{~kg}$ (Tab. 3). La taille des lots visités varie d'un élevage à l'autre. La densité moyenne au premier jour était de 22 poussins par mètre carré. Le taux de mortalité est compris entre 1,5 et $6 \%$.

\subsection{Pratiques d'élevage}

Le questionnaire nous a permis de recueillir des informations sur les pratiques d'élevage et le niveau d'hygiène dans les établissements concernés par l'étude. Nous relevons d'abord que $72 \%$ des élevages visités utilisent une eau provenant de puits et qu'ils ne sont pas contrôlés ou suivis de manière rigoureuse par les services compétents. Les $28 \%$ restants sont liés au réseau de l'Office national de l'eau potable.

Nos résultats montrent également que $68 \%$ des bâtiments visités sont désinfectés une seule fois par bande d'élevage, cette opération étant pratiquée dans la totalité des cas par l'éleveur. Le tiers restant est désinfecté deux fois par bande d'élevage. Dans les cas où il existe plusieurs bâtiments d'élevage par ferme, plus de la moitié des éleveurs pratiquent un vide sanitaire simultané (bâtiment laissé vacant pendant une certaine période), dont la durée moyenne est de 20 jours.

\subsection{Prévalence de Salmonella}

Salmonella a été isolée dans $24 \%$ des élevages examinés (Tab. 4). Pour 12 lots sur les 18 contaminés, la bactérie était présente dans au moins sept pools sur dix analysés.

\subsection{Analyse univariée des facteurs favorisant la contamination par Salmonella}

Les résultats de l'analyse univariée de l'association entre les variables explicatives et la variable «présence/ absence de Salmonella » dans le lot sont résumés dans le Tableau $5.46 \%$ des facteurs testés sont associés à la présence de Salmonella dans les lots en fin de la période d'élevage $(p<0,05)$.

\subsubsection{Facteurs liés aux bâtiments}

Le risque de contamination des lots par Salmonella augmente lorsque la bande d'élevage précédente est contaminée par cette bactérie (Tab. 5). En outre, la prévalence de la bactérie dans les élevages suit une variation saisonnière ( $p<0,047)$, avec un pic de contamination pendant les saisons chaudes $(70 \%$ de contamination pendant les saisons chaudes pour $26,6 \%$ pendant les saisons froides).

\subsubsection{Facteurs liés aux pratiques d'élevage}

Certaines pratiques d'élevage induisent un effet direct ou indirect sur l'infection des poulets par Salmonella. On constate que plus la densité des poussins au premier jour est importante, plus la contamination est fréquente $(p<0,041)$. L'infection des poussins à la mise en place (Tab. 5) a aussi un impact sur l'infection du lot par Salmonella $(p<0,017)$. Par contre, la pratique d'un traitement par antibiotiques au premier jour et au cours de l'élevage s'avère protectrice ( $p<0,034$ et $p<0,044$, respectivement). Nos résultats révèlent également que la proportion d'élevages infectés est près de trois fois plus élevée lorsque les animaux sont âgés de plus de 40 jours au prélèvement $(p<0,017)$, indiquant ainsi un effet de l'âge. En outre, plus la durée du vide sanitaire est longue, moins l'infection est fréquente.

\subsubsection{Facteurs liés aux pratiques d'hygiène}

La présence de la bactérie semble liée aux pratiques sanitaires durant le vide sanitaire précédant la mise en place des poussins : le nettoyage avec des détergents $(p<0,034)$, ainsi que la désinfection des poulaillers $(p<0,041)$ constituent des éléments protecteurs. Au contraire, le stockage du fumier dans la ferme favorise la propagation de la bactérie et son développement dans les exploitations $(p<0,042)$. 
Tableau 2. Définition et distribution des variables explicatives retenues pour l'analyse de l'infection par Salmonella (25 élevages de poulet de chair, Meknès, 2005-2007).

Table 2. Definition and distribution of explanatory variables selected for the analysis of infection by Salmonella (25 broiler-chicken farms, Meknès, 2005-2007).

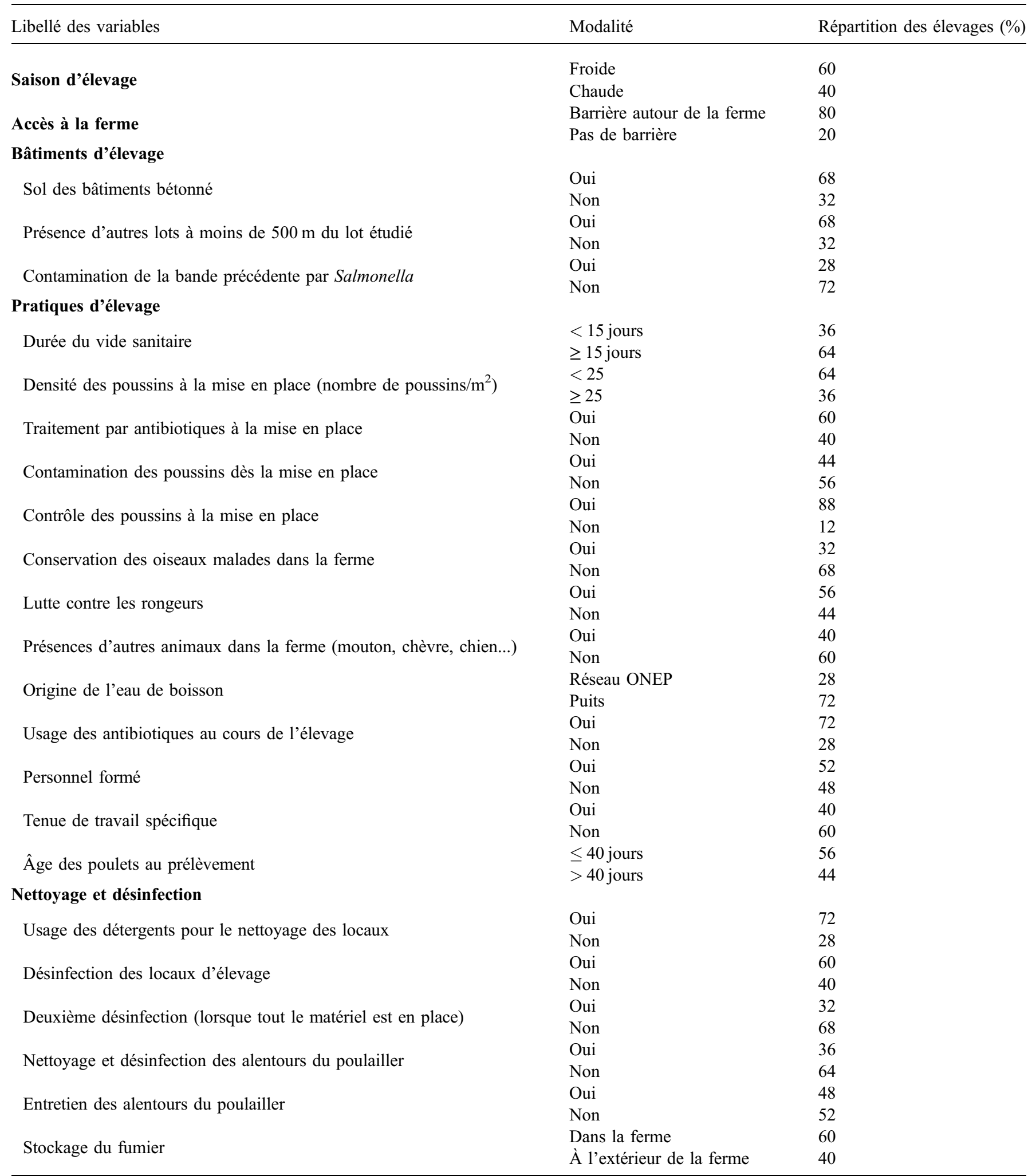

ONEP : Office national de l'eau potable. 
Tableau 3. Caractéristiques des 25 élevages participant à l'étude.

Table 3. Characteristics of the 25 selected farms.

\begin{tabular}{lrr}
\hline Caractéristiques du lot & Minimum & Moyenne \\
\hline Densité des poussins à la mise en place (nombre de poussins $/ \mathrm{m}^{2}$ ) & 12 & 22 \\
Âge à l'abattage (en jour) & 34 & 40 \\
Poids à l'abattage (en g) & 900 & 45 \\
Taille du lot (nombre d'oiseaux) & 450 & 1400 \\
Taux de mortalité & 1,5 & 800 \\
\hline
\end{tabular}

Tableau 4. Répartition (en \%) des lots étudiés en fonction du nombre d'échantillons fécaux contaminés par Salmonella. Table 4. Distribution (in \%) of studied batches depending to the number of faecal samples contaminated by Salmonella.

\begin{tabular}{|c|c|c|c|c|c|c|c|c|c|}
\hline \multirow{4}{*}{$\begin{array}{l}\text { Nombre de pools Salmonella }+ \\
\text { Nombre de lots }\end{array}$} & \multicolumn{8}{|c|}{ Lots Salmonella +} & \multirow{2}{*}{$\frac{\text { Lots Salmonella }}{57 / 75(76 \%)^{\mathrm{b}}}$} \\
\hline & \multicolumn{8}{|c|}{$18 / 75(24 \%)^{\mathrm{a}}$} & \\
\hline & 1 & 2 & 3 & 4 & 5 & 6 & 7 & 8 & 0 \\
\hline & 0 & 3 & 0 & 0 & 3 & 0 & 8 & 4 & 57 \\
\hline
\end{tabular}

${ }^{\mathrm{a}}$ Taux de lots salmonella + .

${ }^{\mathrm{b}}$ Taux de lots salmonella -.

\section{Discussion}

L'étude réalisée a permis d'identifier les facteurs favorisant l'infection des élevages de poulets de chair par la bactérie Salmonella dans la région de Meknès (Maroc), ainsi que leur importance relative. Cette information permet d'étudier les marges de manœuvre pour réduire la contamination des élevages, dans le but d'élaborer des stratégies de contrôle ciblées.

Salmonella a été isolée dans $24 \%$ des élevages visités. Pour plus de $66 \%$ de cas contaminés, on trouvait la bactérie dans plus de six sur dix pools de fientes récoltés, confirmant ainsi l'importance de la contamination horizontale au sein d'une bande d'élevage (Bailey et al., 2002 ; Gradel et Rattenborg, 2003). Au Maroc, aucune étude relative à la prévalence de Salmonella dans les élevages n'a encore été publiée. Dans les pays en développement, les rares études disponibles révèlent $100 \%$ d'infection en Thaïlande (Sasipreeyajan et al., 1996), 28,6 \% au Sénégal (Cardinale et al., 2004) et 21,7\% au Brésil (Tavechio et al., 2002). Dans les pays développés, la prévalence de Salmonella dans les élevages de poulet de chair varie d'un pays à l'autre : 76,9 \% au Canada (Chambers et al., 1998) ; 69,8 \% en France (Rose et al., 1999) ; 41,3\% en Turquie (Carli et al., 2001) ; $25 \%$ au Danemark (Chadfield et al., 2001) ; 0,7 \% en Finlande et $0,03 \%$ en Suède (Anon, 1998). En revanche, il faut reconnaître que différentes méthodes de prélèvement sont utilisées dans les différentes études; la comparaison de ces données doit donc être effectuée avec précaution.

La saison s'est avérée significativement associée à la contamination des élevages $(\mathrm{OR}=6,42)$. En effet, seuls quatre sur 15 élevages visités pendant la saison froide étaient contaminés, alors que sept parmi les dix élevages visités pendant la saison chaude, étaient contaminés (Tab. 6). Il est évident que la saison chaude offre des conditions de chaleur et d'humidité qui sont favorables au développement des salmonelles. Au contraire, le froid peut ralentir la multiplication de ces bactéries.
La densité des poussins dans le bâtiment $(\mathrm{OR}=7,7)$ montre une association statistiquement significative avec les contaminations par les salmonelles. Les élevages de poulet de chair où la densité est en dessous de 25 sujets par mètre carré ont un taux de contamination plus faible que celui des élevages de densité supérieure à 25 sujets par mètre carré. Ces résultats sont en accord avec la littérature qui rapporte que la forte densité dans un élevage de poulets est un facteur favorisant la contamination par les salmonelles (Heyndrickx et al., 2002).

Nos résultats montrent que la contamination de la bande d'élevage précédente semble augmenter significativement le risque de contamination par Salmonella spp. $(\mathrm{OR}=5,14)$. Quatre élevages sur sept dont la bande précédente était infectée sont également infectés. Ce constat correspond bien aux résultats relevés par Bailey et al. (2002) et par Gradel et Rattenborg (2003). Les sérotypes isolés dans les bandes précédentes sont souvent isolés également dans les nouveaux lots à l'enlèvement (Gradel et Rattenborg, 2003). Selon Lahellec et al. (1986), même si le poussin arrive exempt de salmonelles, une infection reste possible avec un bâtiment mal nettoyé et mal désinfecté, malgré un vide sanitaire.

Les poussins déjà contaminés dès leur arrivée dans l'élevage sont un facteur de risque important $(\mathrm{OR}=10,5)$. Ils contribuent à l'augmentation du niveau de contamination des bâtiments d'élevage par l'intermédiaire de leurs déjections (Colin, 1992). Ce constat confirme les études de Rose et al. (1999) et de Christensen et al. (1997).

L'utilisation des antibiotiques dès le premier jour $(\mathrm{OR}=$ 9,33 ) est un moyen prophylactique de lutte contre la contamination par Salmonella des oiseaux en élevage (Chriel et al., 1999).

Le risque de contamination du lot par Salmonella décroît avec la durée du vide sanitaire $(\mathrm{OR}=8,67)$. Ce risque est significativement réduit lors d'un vide sanitaire de plus de 15 jours. Enfin, la probabilité de contamination augmente avec l'âge des poulets au prélèvement $(\mathrm{OR}=9,78)$. Ainsi, les 
Tableau 5. Analyse de l'association entre les variables explicatives et la variable « présence/absence de Salmonella » dans le lot étudié à la fin de la période d'élevage (test du $\mathrm{Khi}^{2}$ à $5 \%$ ).

Table 5. Analysis of the links between explanatory variables and "presencelabsence of Salmonella" in the batch studied at the end of the breeding period (5\% Khi ${ }^{2}$ test).

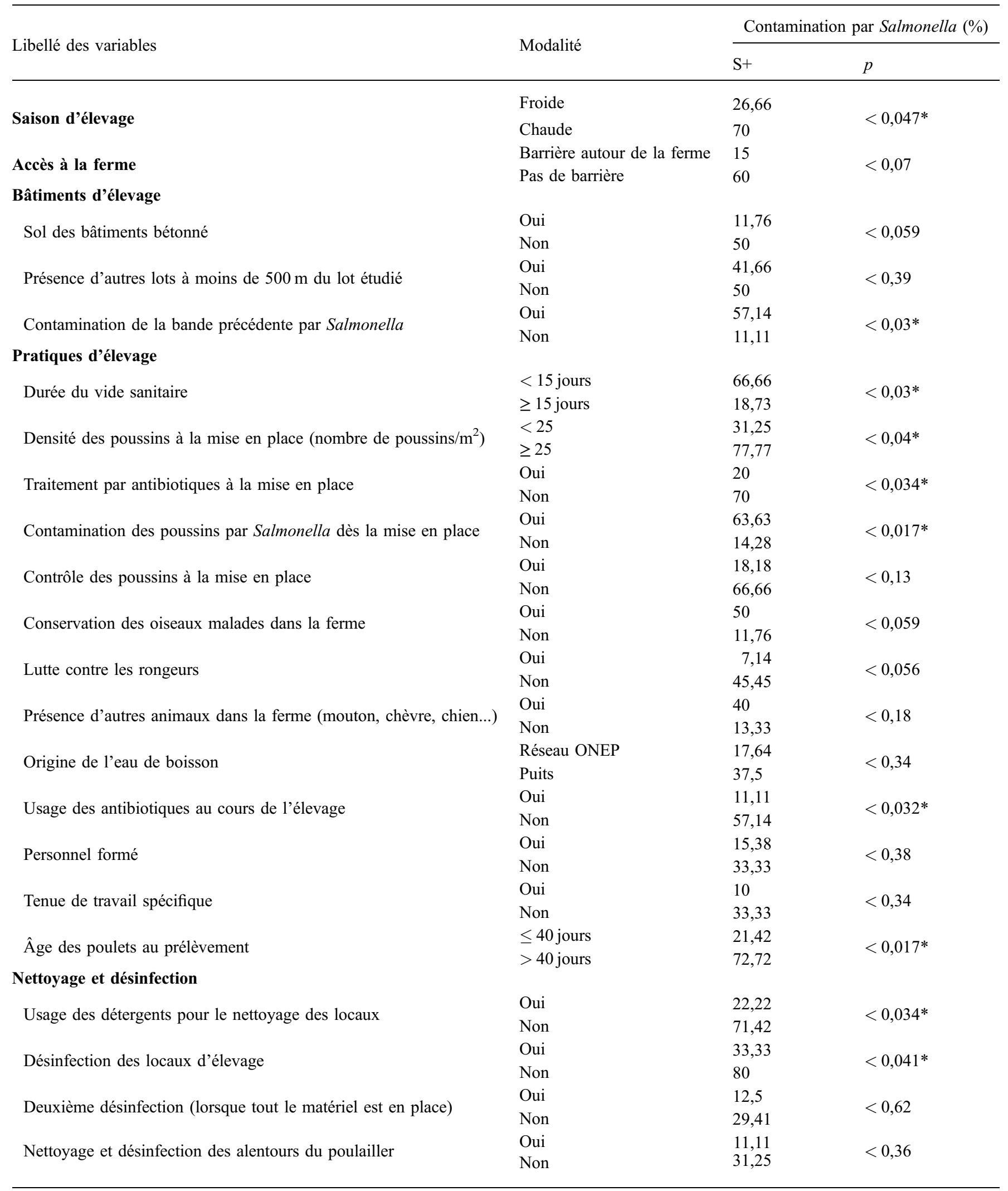


Tableau 5. (suite).

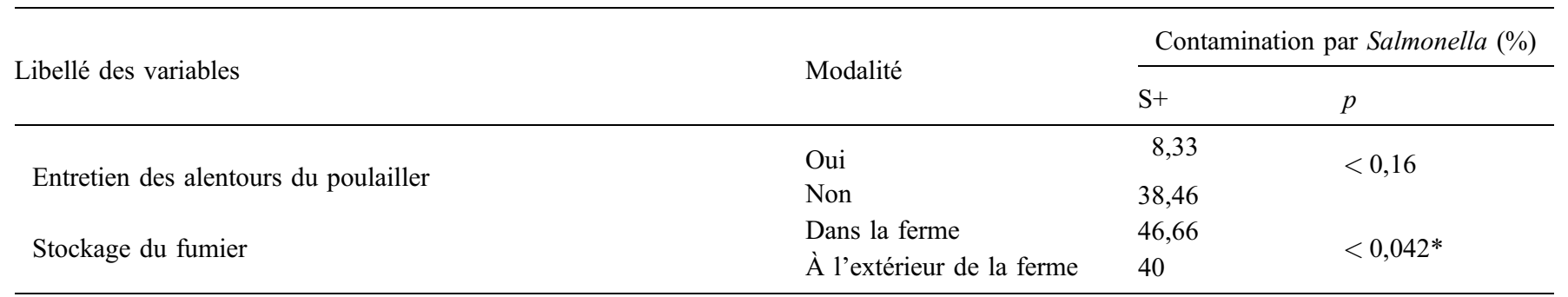

${ }^{*} p<0,05$ : variable significativement associée à l'infection par Salmonella ; S + : infection par Salmonella ; ONEP : Office national de l'eau potable.

Tableau 6. Facteurs associés à la contamination des 25 élevages de poulets de chair par Salmonella spp. $(n=25)$.

Table 6. Factors associated with the contamination of the 25 broiler-chicken farms by Salmonella spp.

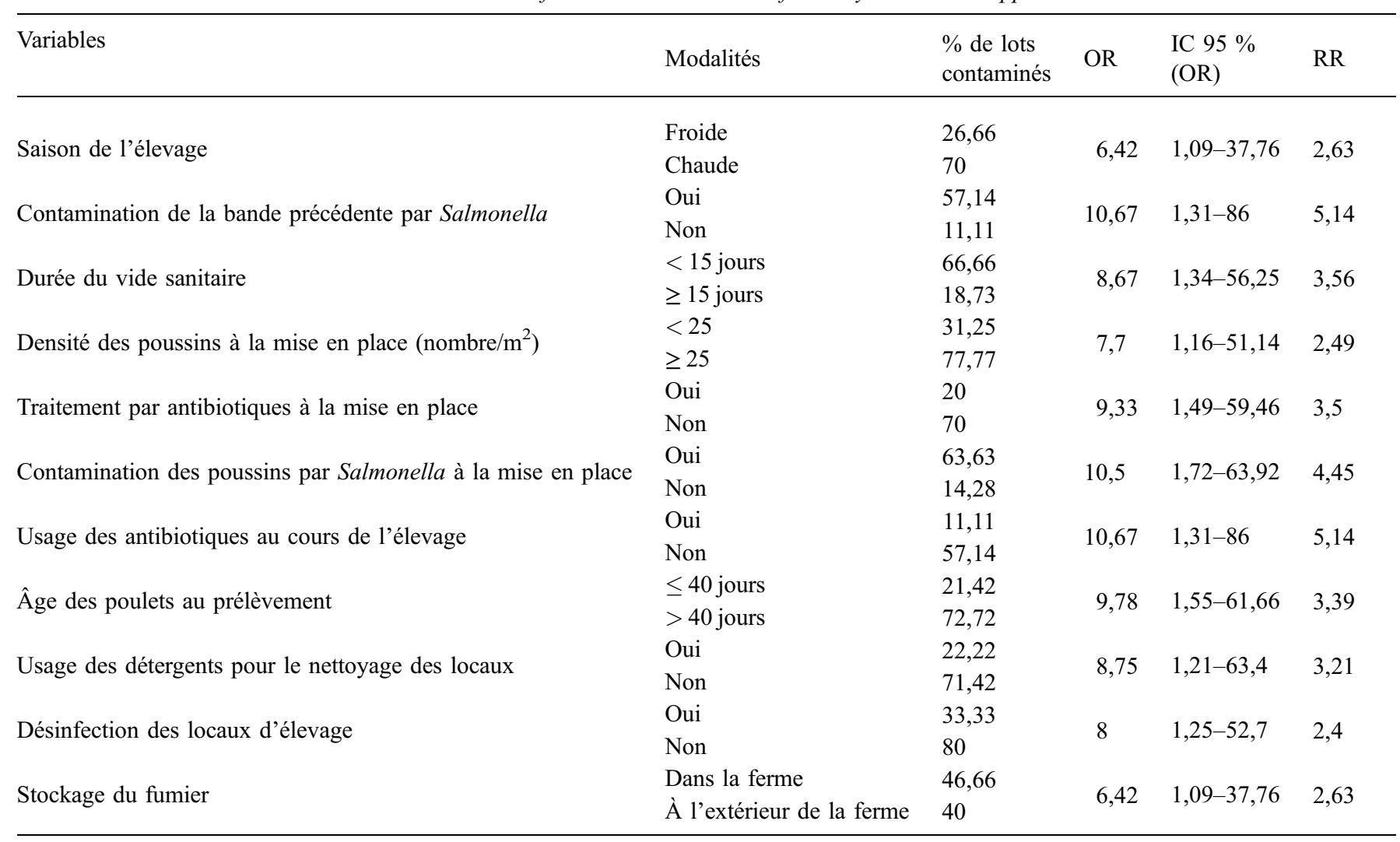

OR : odds ratio (rapport des cotes) ; IC $95 \%$ (OR) : intervalle de confiance pour odds ratio à $95 \%$ selon la méthode Woolf (méthode des logits) ; RR : risque relatif.

poulets sont davantage sujets à la contamination après 40 jours. Ce résultat est soutenu par différentes hypothèses : la longueur de la durée de la bande d'élevage engendre plus de passages dans le bâtiment et constitue par conséquent un vecteur potentiel de contamination par Salmonella (Cardinale et al., 2004).

D'autres études montrent par ailleurs que tout intrant dans l'élevage est susceptible de véhiculer la bactérie d'unités infectées à d'autres. Tel est le cas du personnel en l'absence de tenue spécifique (Cardinale et al., 2004) ou des visiteurs étrangers à la ferme (Fris et Van Den Bos, 1995). Il en est de même pour les véhicules circulant autour du bâtiment.
Le non-respect de certaines pratiques d'hygiène favorise la présence de Salmonella dans les élevages. Les résultats révèlent en effet que le nettoyage et la désinfection sont des facteurs critiques, dans la mesure où le pourcentage de contamination est réduit lorsque ces opérations ont été pratiquées régulièrement (Angen et al., 1996). La contamination fécale de la litière est par ailleurs considérée comme une des sources les plus probables d'infection des jeunes troupeaux dans des bâtiments nettoyés de façon imparfaite ou contenant de la litière recyclée (Line, 2002).

Il est donc fondamental de maintenir des règles strictes de biosécurité dans l'élevage, avec l'utilisation d'une litière de 
bonne qualité et son renouvellement entre chaque lot afin de prévenir l'infection d'un lot à un autre par Salmonella.

Nous avons observé que la prévalence de Salmonella est faible dans les élevages avicoles qui utilisent une eau du réseau de l'Office national de l'eau potable. Ce constat est en accord avec les résultats de Tablante (2002), qui rapporte que le traitement de l'eau réduit le nombre de germes pathogènes présents dans l'eau de boisson, il est donc un facteur protecteur de la contamination des poulets de chair par les pathogènes.

\section{Conclusion}

Le taux de contamination par Salmonella des élevages de poulet de chair dans la région de Meknès est élevé. Notre étude a permis de déterminer les facteurs potentiellement associés à cette contamination. Ces facteurs rejoignent ceux souvent rapportés dans la littérature internationale, mais c'est la première fois que ce type de données est disponible au Maroc.

Des mesures permettraient de réduire la contamination dans les élevages de poulets et tout au long de la chaîne de production. Il est en effet fondamental de respecter certaines pratiques d'hygiène telles qu'un nettoyage-désinfection efficace dans le bâtiment, accompagné d'un changement de litière et d'un vide sanitaire de durée conséquente.

Une grande attention doit être portée à l'eau, potentiel vecteur de contaminants. Sa qualité microbiologique doit être régulièrement contrôlée. De plus, si les poulets sont porteurs de la bactérie pendant l'élevage, la transmission est possible à tous les stades de la chaîne de production. Ainsi, le consommateur doit lui aussi être acteur de cette réduction de la fréquence de Salmonella, en respectant les mesures d'hygiène.

\section{Références}

Angen O, Skov MN, Chriel M, Agger JF, Bisgaard M. 1996. A retrospective study on Salmonella infection in Danish broiler flocks. Prev. Vet. Med. 26: 223-237.

Anon A. 1998. Salmonella in livestock production. Weybridge: Veterinary Laboratories Agency, Ministry of Agriculture, Fisheries and Food, Welsh Office Agriculture Department, Scottish Office Agriculture and Fisheries Department.

Bailey JS, Stern NJ, Fedorka-Cray P, et al. 2001. Sources and movement of Salmonella through integrated poultry operations: a multistate epidemiological investigation. J. Food Prot. 64(11): 1690-1697.

Bailey JS, Cox NA, Craven SE, Cosby DE. 2002. Serotype tracking of Salmonella through integrated broiler-chicken operations. J. Food Prot. 65: 742-745.

Cardinale E, Tall F, Guèye EF, Cisse M, Salvat G. 2004. Risk factors for Salmonella enterica subsp. enterica infection in Senegalese broiler-chicken flocks. Prev. Vet. Med. 63: 151-161.

Carli KT, Eyigor A, Caner V. 2001. Prevalence of Salmonella serovars in chicken in Turkey. J. Food Prot. 64(11): 1832-1835.
Chadfield M, Skov M, Christensen J, Madsen M, Bisgaard M. 2001. An epidemiological study of Salmonella enterica serovar 4, 12:b: in broiler-chicken in Denmark. Vet. Microbiol. 82(3): 233-247.

Chambers JR, Bisaillon JR, Labbe Y, Poppe C, Langford CF. 1998. Salmonella prevalence in crops of Ontario and Quebec broilerchicken at slaughter. Poult. Sci. 77(10): 1497-1501.

Chriel M, Stryhn H, Dauphin G. 1999. Generalised linear mixed models analysis of risk factors for contamination of Danish broiler flocks with Salmonella typhimurium. Prev. Vet. Med. 40(1): $1-17$.

Christensen JP, Brown DJ, Madsen M, Olsen JE, Bisgaard M. 1997. Hatchery-borne Salmonella enterica serovar Tennessee infections in broilers. Avian Pathol. 26: 155-168.

Colin P. 1992. Salmonella et qualité des produits avicoles. In: Brugere-Picoux J, Silim A, eds. Manuel de pathologie aviaire. Édition Chaire de pathologie médicale du bétail et des animaux de basse-cour. Alfort: École nationale vétérinaire d'Alfort, 371-373.

Davies R, Breslin M, Corry JE, Hudson W, Allen VM. 2001. Observations on the distribution and control of Salmonella species in two integrated broiler companies. Vet. Rec. 149(8): 227-232.

Fris C, Van Den Bos J. 1995. A retrospective case-control of risk factors associated with Salmonella enterica subsp. enterica serovar enteritidis infections on Dutch broiler breeder farms. Avian Pathol. 24: $255-272$.

Gradel KO, Rattenborg EA. 2003. Questionnaire-based retrospective field study of persistence of Salmonella enteritidis and Salmonella typhimurium in Danish broiler houses. Prev. Vet. Med. 56: 267-284.

Heyndrickx M, Vandekerchove D, Herman L, Rollier I, Grijspeerdt K, De Zutter L. 2002. Routes for Salmonella contamination of poultry meat: epidemiological study from hatchery to slaughterhouse. Epidemiol. Infect. 129: 253-265.

Kimura C, Reddy V, Marcus R, et al. 2004. Chicken consumption is a newly identified risk factor for sporadic Salmonella enterica serotype Enteritidis infections in the United States, a case-control study in FoodNet sites. Clin. Infect. Dis. 38: 244-252.

Lahellec C, Colin P, Bennejean G, Paquin J, Guillerm A, Debois JC. 1986. Influence of resident Salmonella on contamination of broiler flocks. Poult. Sci. 65(11): 2034-2039.

Line JE. 2002. Campylobacter and Salmonella populations associated with chickens raised on acidified litter. Poult. Sci. 81: 1473-1477.

Mead GC. 1993. Problems of producing safe poultry: discussion paper. J. R. Soc. Med. 86: 39-42.

Rose N, Beaudeau F, Drouin P, Toux JY, Rose V, Colin P. 1999. Risk factors for Salmonella enterica subsp. enterica contamination in French broiler-chicken flocks at the end of the rearing period. Prev. Vet. Med. 39(4): 265-277.

Sasipreeyajan J, Jerngklinchan J, Koowatananukul C, Saitanu K. 1996. Prevalence of Salmonella in broiler. Trop. Anim. Health Prod. 28(2): $174-180$.

Tablante NL. 2002. A survey of biosecurity practices as risk factors affecting broiler performance on the Delmarva Peninsula. Avian Dis. 46: 730-734.

Tavechio AT, Ghilardi AC, Peresi JT, et al. 2002. Salmonella serotypes isolated from nonhuman sources in Sao Paulo Brazil from 1996 through 2000. J. Food Prot. 65(6): 1041-1044.

Thorns CJ. 2000. Zoonoses bactériennes d'origine alimentaire. Rev. Sci. Tech. 19: 226-239.

Cite this article as: Chaiba A, Rhazi Filali F. 2016. Prévalence de la contamination par Salmonella des élevages de poulet de chair au Maroc. Cah. Agric. 25: 35007. 